\title{
Overexpression of Arabidopsis Laccase2, Laccase4 and Peroxidase52 in hybrid poplar under developing xylem specific DX15 promoter improves saccharification efficiency in a woody biofuel feedstock
}

\author{
YOGESH AHLAWAT *, Chandrashekhar P Joshi *
}

\begin{abstract}
Laccases (LAC) and Peroxidases (PRX) are the key enzymes responsible for the polymerization of a variety of monolignols into lignin. However, little is known about the functions of various members of Lac and Prx gene families in plants. The goal of this research is to understand the biological functions of LAC and PRX in poplar wood formation and to explore how modified Lac and Prx expression affects biomass recalcitrance and growth. This study of heterologous expression of Arabidopsis Lac and Prx genes was conducted in poplars to mitigate the occurrence of co-suppression commonly occurs during the homologous overexpression of highly expressed native genes. To this end, we overexpressed selected Arabidopsis laccase2 (AtLac2), laccase4 (AtLac4), and peroxidase52 (AtPrx52) genes, based on their high level of transcript expression in the differentiating xylem tissues of stems, in hybrid poplar under the developing xylem tissue-specific promoter, DX15 and characterized the transgenic plants for growth phenotypes and recalcitrance efficiency. Transgenic poplar plant lines overexpressing the AtLac2 gene (AtLac2-OE) showed an increase in plant height without a change in biomass yield as compared to the controls. Whereas, AtLac4-OE and AtPrx52-OE transgenic lines did not show any such observable growth phenotypes compared to their respective controls. The total xylem area was decreased by $26 \%$ in AtPrx $52-\mathrm{OE}$ lines and by $6 \%$ in AtLac4-OE lines whereas, the xylem area increased by about $8 \%$ in AtLac2-OE lines compared to the controls. Total lignin content was decreased by $2-6 \%$ in all the transgenic lines than the controls. This led to an increase in the $\mathrm{S} / \mathrm{G}$ ratio by $3-10 \%$ in all transgenic lines (AtLac2$\mathrm{OE}$, AtLac4-OE, and AtPrx52-OE) as compared to the controls. The changes in lignin content and $\mathrm{S} / \mathrm{G}$ ratios in the transgenic poplar lines resulted in a significant increase in the saccharification efficiency as compared to the control plants. Overall, saccharification efficiency was increased by $35-50 \%, 21-42 \%$, and $8-39 \%$ in AtLac2-OE, AtLac4-OE, and AtPrx52-OE transgenic poplar lines respectively, as compared to their controls. Therefore, heterologous expression of specific AtLac and AtPrx genes in poplar species could be one of the potential strategies for increasing saccharification efficiency in woody feedstock for the improved production of bioethanol.
\end{abstract}

Keywords: cell wall; secondary cell wall; saccharification; biofuel 